\title{
Relação entre níveis de chumbo, condições periodontais e qualidade de vida entre trabalhadores de uma fábrica de baterias automotivas no município de Bauru - SP
}

\author{
Relationship between lead levels, periodontal conditions and \\ quality of life among workers at an automotive battery factory \\ in Bauru - SP county
}

\section{Relación entre niveles de plomo, condiciones periodontales y calidad de vida entre trabajadores de una fábrica de baterias automotivas en el município de Bauru - SP}

Adriana Rodrigues de Freitas Aznar iD

Fábio Duarte da Costa Aznar iD

Priscila Matter Borges ${ }^{1}$

José Burgos Ponce ic

Arsenio Sales-Peres iD

1 priscila.borges@usp.br

Endereço para correspondência:

Adriana Rodrigues de Freitas Aznar

Faculdade de Odontologia de Bauru - USP

Departamento de Odontopediatria, Ortodontia e Saúde Coletiva

Alameda Doutor Octávio Pinheiro Brisolla, 9-75

17012-901 - Bauru - São Paulo - Brasil

E-mail: adrianafreitas@usp.br

Recebido: 25.08 .2020

Aceito: 01.09.2020

\section{RESUMO}

Objetivou-se analisar a relação entre os níveis sanguíneos de chumbo, as condições periodontais e o impacto bucal na qualidade de vida entre trabalhadores de uma fábrica de baterias automotivas no município de Bauru - SP. A amostra foi composta por 70 trabalhadores, avaliados por examinador calibrado (Kappa $\geq 0.78$ ), registrando-se os níveis sanguíneos de chumbo (NSPB), hábitos de vida, higiene bucal e impacto bucal na qualidade de vida (OHIP-14). Utilizou-se Índice Periodontal Comunitário (IPC) e Índice de Placa (IP). Adotou-se nível de significância 5\% $(p<0.05)$, sendo aplicados teste t de Student, Mann-Whitney, Odds Ratio, Qui-Quadrado e correlação linear de Pearson. A idade média foi de $33.21 \pm 9.43$ anos, $88.57 \%$ eram do sexo masculino, $12.85 \%$ hipertensos, $10 \%$ tabagistas, $12.86 \%$ consumiam regularmente bebidas alcoólicas, $88.57 \%$ escovavam os dentes 3 ou mais vezes/dia, $97.14 \%$ uso frequente de fio dental. O nível médio de chumbo no sangue $44.01 \pm 6.15 \mu \mathrm{g} / \mathrm{dL}$, sendo maiores entre trabalhadores da linha de produção $(\mathrm{p}=0.000)$. Observaram-se alterações periodontais em $67.14 \%$ dos indivíduos, com sangramento (25.71\%), cálculo $(67.14 \%)$, bolsas rasas $(20.00 \%)$ e bolsas 
profundas $(10.00 \%)$. NSPB $>40 \mu \mathrm{g} / \mathrm{dl}$ estiveram associados ao sangramento gengival (OR $=4.91 ; p=0.006)$. O IP foi $2.78 \pm$ 0.54. O impacto bucal foi baixo, $4.48 \pm 1.87$, havendo correlação positiva entre os NSPB e a incapacidade física $(\mathrm{r}=0.35 ; p=$ $0.003)$, e dos escores totais OHIP-14 com IP $(r=0.24 ; p=0.044)$. Embora nenhum dos trabalhadores tenha apresentado NSPB acima do Índice Máximo Biológico Permitido, a prevalência de alterações periodontais encontrada foi alta, sendo relacionadas à pobre higiene bucal e evidenciando a necessidade de adoção de medidas terapêuticas, preventivas e educativas.

PALAVRAS-CHAVE: Doenças periodontais. Intoxicação por chumbo. Qualidade de vida.

\begin{abstract}
The objective was to analyze the relationship between blood lead levels, periodontal conditions and the oral impact on quality of life among workers at an automotive battery factory in the city of Bauru - SP. The sample consisted of 70 workers, assessed by a calibrated examiner (Kappa $\geq 0.78$ ), recording blood lead levels (NSPB), lifestyle, oral hygiene and oral impact on quality of life (OHIP-14). Community Periodontal Index (CPI) and Plaque Index (PI) were used. The level of significance was set at $5 \%(\mathrm{p}<0.05)$, the Student's t-test, Mann-Whitney, Odds Ratio, Chi-Square and Pearson's linear correlation being applied. The average age was $33.21 \pm 9.43$ years, $88.57 \%$ were male, $12.85 \%$ were hypertensive, $10 \%$ smokers, $12.86 \%$ regularly consumed alcoholic beverages, $88.57 \%$ brushed their teeth 3 or more times/day, $97.14 \%$ frequent flossing. The average level of lead in the blood $44.01 \pm 6.15 \mu \mathrm{g} / \mathrm{dL}$, being higher among workers of the production line $(\mathrm{p}=0.000)$. Periodontal changes were observed in $67.14 \%$ of the people envolved, with bleeding $(25.71 \%)$, calculus $(67.14 \%)$, shallow bags $(20.00 \%)$ and deep bags $(10.00 \%)$. NSPB $>40 \mu \mathrm{g} / \mathrm{dl}$ were associated with gingival bleeding $(\mathrm{OR}=4.91 ; \mathrm{p}=0.006)$. The IP was $2.78 \pm 0.54$. The oral impact was low, $4.48 \pm 1.87$, with a positive correlation between NSPB and physical disability ( $\mathrm{r}=0.35 ; \mathrm{p}=0.003)$, and the total OHIP-14 scores with PI $(r=0.24 ; p=0.044)$. Although none of the workers had NSPB above the Maximum Biological Allowable Index, the prevalence of periodontal changes found was high, being related to poor oral hygiene and highlighting the need to adopt therapeutic, preventive and educational measures.
\end{abstract}

KEYWORDS: Periodontal diseases. Lead poisoning. Quality of life.

\title{
RESUMEN
}

El objetivo fue analizar la relación entre los niveles de plomo en sangre, las condiciones periodontales y el impacto bucal en la calidad de vida entre los trabajadores de una fábrica de baterías automotivas en el municipio de Bauru - SP. La muestra fue constituida por 70 trabajadores, evaluados por un examinador calibrado (Kappa $\geq 0.78$ ), registrando los niveles sanguíneos de plomo (NSPB), hábitos de vida, higiene e impacto bucales en la calidad de vida (OHIP-14). Se utilizó el Índice Periodontal Comunitario (IPC) e Índice de Placa (IP). Se adoptó un nivel de significancia de 5\% (p $<0.05)$, aplicando test t de Student, Mann-Whitney, Odds Ratio, Chi-cuadrado y correlación lineal de Pearson. La edad media fue de $33.21 \pm 9.43$ años, $88.57 \%$ correspondían al sexo masculino, $12.85 \%$ hipertensos, $10 \%$ fumadores, $12.86 \%$ consumían regularmente bebidas alcohólicas, $88.57 \%$ cepillaban los dientes 3 o más veces/dia, $97.14 \%$ uso frecuente de hilo dental. El nivel medio de plomo en sangre 44.01 $\pm 6.15 \mu \mathrm{g} / \mathrm{dL}$, siendo mayores entre trabajadores dentro de la línea de producción $(\mathrm{p}=0.000)$. Fueron observadas alteraciones periodontales en $67.14 \%$ de los individuos, con sangrado $(25.71 \%)$, cálculo $(67.14 \%)$, bolsas rasas $(20.00 \%)$ y bolsas profundas $(10.00 \%)$. NSPB $>40 \mu \mathrm{g} / \mathrm{dl}$ estuvieron asociados al sangrado gingival $(\mathrm{OR}=4.91 ; p=0.006)$. El IP fue de $2.78 \pm 0,54$. El impacto bucal fue bajo, $4.48 \pm 1.87$, existiendo correlación positiva entre los NSPB y la incapacidade física $(\mathrm{r}=0.35 ; p=0.003)$, $\mathrm{y}$ los valores totales OHIP-14 con IP $(\mathrm{r}=0.24 ; \mathrm{p}=0.044)$. Si bien ninguno de los trabajadores presentó NSPB por encima del Índice Biológico Máximo Permitido, la prevalencia de alteraciones periodontales encontradas fue alta, estando relacionada con la mala higiene bucal y destacando la necesidad de la adopción de medidas terapéuticas, preventivas y educativas.

PALABRAS CLAVE: Enfermedades periodontales. Intoxicación por plomo. Calidad de vida. 


\section{INTRODUÇÃO}

A Odontologia do Trabalho é a especialidade que tem como objetivo a busca permanente da compatibilidade entre a atividade laboral e a preservação da saúde bucal do trabalhador'. Assim sendo, torna-se de fundamental importância o conhecimento do profissional odontólogo que trabalha com esta área, de estar bem preparado para analisar as causas e efeitos das manifestações bucais decorrente do processo laboral, bem como instituir medidas preventivas e orientar tratamentos.

Inúmeras são as enfermidades que podem acometer o sistema estomatognático devido às ocupações laborais. Em praticamente todas as atividades ocupacionais existem riscos, com classificações que variam dependendo do próprio ambiente de trabalho. Esses podem agir isolados ou conjuntamente, e são representados pela ação de agentes físicos, mecânicos, químicos, biológicos ou ergonômicos e psicossociais ${ }^{2}$.

Dentre as formas de contaminação química que pode acometer os trabalhadores, uma delas é através do contato inadequado com o chumbo, sendo que no Brasil, os maiores consumidores deste material são as indústrias de baterias automotivas, responsáveis por cerca de $80 \%$ do uso deste metal $^{3}$.

Os trabalhadores que se encontram na linha de produção destas empresas que utilizam chumbo como matéria-prima estão mais suscetíveis á intoxicações por este metal devido a um maior tempo de exposição ocupacional. Este metal pode penetrar o organismo através da inalação (ar atmosférico), ingestão (água, alimentos e solo contaminados) e por via dérmica. As principais manifestações clínicas pela exposição ao chumbo são gastrintestinais, renais, reprodutivas, hematológicas, neurológicas e carcinogênicas ${ }^{4}$.

Assim que é absorvido, o chumbo é distribuído para o sangue, com uma meia-vida de 37 dias; para os tecidos moles, com uma meia-vida de 40 dias; e para os ossos, onde possui uma meia-vida de 27 anos, constituindo este o seu maior depósito corporal representado por 90 a $95 \%$ de todo o chumbo presente no corpo ${ }^{5}$.

Estudos apontam que a exposição crônica de chumbo pode afetar o metabolismo do osso ${ }^{6.7}$ e, possivelmente, o sistema imunológico ${ }^{8.9}$. Esta evidência mostra um potencial risco para a periodontite, uma doença multifatorial, iniciada por um desequilíbrio do sistema de defesa do hospedeiro e a patogenicidade de microorganismos ${ }^{10-11}$. Quando substâncias químicas em doses elevadas são inaladas ou absorvidas, elas irão apresentar alterações no fluxo salivar e na composição bioquímica da saliva, acarretando em um aumento do número de lesões cariosas e no desenvolvimento da doença periodontal ${ }^{12}$.

Os limites de tolerância biológicos propostos para a intoxicação ocupacional pelo metal variam de legislação de acordo com cada país. A American Conference of Government Industrial Hygienists dos Estados Unidos, apresenta como índice biológico de exposição o valor de 30 $\mu \mathrm{g} / \mathrm{dL}^{13}$. No Brasil, a NR-7 (Norma Regulamentadora n. ${ }^{0}$ 7) é instituída com a finalidade de orientar a operacionalização do Programa de Controle Médico de Saúde Ocupacional $(\mathrm{PCMSO})^{14}$, sendo que a mesma indica que o valor de referência da normalidade (valor encontrado em população não exposta) para o chumbo no sangue é de $40 \mu \mathrm{g} / \mathrm{dL}$, e o Índice Máximo Biológico Permitido (IMBP) é de $60 \mu \mathrm{g} / \mathrm{dL}$. Quando este valor é ultrapassado indica exposição excessiva do trabalhador ao metal, causando possíveis riscos à saúde $\mathrm{e}^{15}$.

De acordo com o Ministério da Saúde, um trabalhador com sintomatologia de doença ocupacional aponta a existência de outros casos dentro do local e necessidade de melhoria no ambiente de trabalho. Portanto, pela possibilidade de contaminação por este metal, é fundamental e obrigatório o controle periódico e permanente dos níveis de chumbo nos trabalhadores, sendo esta análise realizada através de exame sanguíneo, sendo os níveis, portanto mensurados e documentados no prontuário do trabalhador ${ }^{15}$.

$\mathrm{Na}$ literatura, não existem estudos que analisam a condição periodontal fazendo uma correlação com o nível sanguíneo de chumbo; podendo ser, portanto, um dado importante no planejamento preventivo e de tratamento dos trabalhadores pela Odontologia do Trabalho.

O presente estudo teve como objetivo analisar a relação entre os níveis sanguíneos de chumbo, as condições periodontais e o impacto bucal na qualidade de vida entre trabalhadores de uma fábrica de baterias automotivas no município de Bauru - SP.

\section{MATERIAL E MÉTODOS}

Em respeito à Resolução CNS/MS 466/2012, este estudo foi submetido e aprovado pelo Comitê de Ética em Pesquisa em Seres Humanos da Faculdade de Odontologia de Bauru da Universidade de São Paulo.

O cálculo amostral foi realizado utilizando-se a diferença entre duas proporções, com efeito de desenho de $5 \%$ e erro de $20 \%$. Foram utilizados os dados do Levantamento Nacional de Saúde Bucal "SB Brasil 2010" relativos à prevalência de bolsas periodontais (rasas e profundas, $19.4 \%$ ) na população adulta (35-44 anos) $)^{16}$ e dados sobre a prevalência de contaminação por chumbo (43.4\%), em trabalhadores de fábricas e de reciclagem de baterias automotivas ${ }^{15}$. Utilizando-se estes parâmetros obteve-se amostra de 68 indivíduos, segundo o efeito de desenho, sendo considerados para avaliação 70 trabalhadores. 
Neste setor se faz necessário o controle periódico e permanente dos níveis de chumbo nos trabalhadores, dada a possibilidade de contaminação por este metal pesado. Nesta empresa os exames foram realizados em laboratório de análises clínicas, gerenciados pela equipe do Serviço Especializado em Engenharia e Medicina do Trabalho (SESMT). A análise foi realizada através de exame sanguíneo conforme a NR-7 (Norma Regulamentadora n. ${ }^{\circ}$ $7)^{14}$, sendo que o limite superior de normalidade legal é de 40 $\mu \mathrm{g} / \mathrm{dl}$ e o Índice Biológico Máximo Permitido (IBPM) de 60 $\mu \mathrm{g} / \mathrm{dl}^{15}$. Os valores aferidos são registrados em prontuários como parte da rotina de vigilância à saúde dos trabalhadores, de onde foram coletados os dados utilizados neste estudo.

Este estudo observacional de corte transversal foi constituído por três etapas: calibração do examinador, realização dos exames clínicos e aplicação de questionários. A avaliação das condições periodontais dos indivíduos foi realizada por um único examinador, devidamente calibrado, e seguindo as recomendações da Organização Mundial da Saúde para levantamentos epidemiológicos em saúde bucal $^{17}$. O processo de calibração do examinador foi conduzido por um examinador padrão, experiente em levantamentos epidemiológicos. O coeficiente Kappa intra examinador foi calculado, com o re-teste em $10 \%$ da $\operatorname{amostra}^{18}$, sendo obtido coeficiente de 0.82 para índice de placa, e 0.78 para a presença de bolsas periodontais.

Os exames avaliaram a presença de sangramento gengival, cálculo e bolsas periodontais, utilizando-se sonda periodontal IPC (Índice Periodontal Comunitário), que possui esfera de $0.05 \mathrm{~mm}$ em sua extremidade e graduações de $3.5 \mathrm{~mm}, 5.5 \mathrm{~mm}, 8.5 \mathrm{~mm}$ e $11.5 \mathrm{~mm}^{17}$. Como prérequisito ao exame o sextante deveria possuir dois ou mais dentes sem indicação de exodontia (por exemplo: mobilidade, comprometimento de furca etc.), sendo codificada a situação de maior severidade encontrada.

A presença de placa foi avaliada utilizando-se o Índice de Placa de Turesky, uma modificação do índice proposto por Quigley e $\operatorname{Hein}^{19-20}$. Previamente às avaliações os indivíduos foram submetidos à evidenciação de placa bacteriana, por meio de bochechos com solução de fucsina básica (Eviplac, Biodinâmica, Brasil), dispensada em copos plásticos descartáveis e de acordo com as instruções fornecidas pelo fabricante. Os exames foram realizados utilizando-se espelho bucal plano e espátula de madeira, e foram avaliadas as superfícies vestibular e lingual de cada dente e em seguida atribuindo escores de 0 a 5 . O escore final de cada indivíduo foi obtido por meio da soma de todos os escores de placa obtidos dividido pela quantidade de faces examinadas. A prevalência de placa foi considerada baixa com valores entre $0<2$, média entre $2<4$, e alta $>4$. Após a avaliação os indivíduos foram submetidos à profilaxia profissional utilizando-se taça de borracha, pasta profilática e fio dental, bem como receberam orientações sobre higiene bucal.
Os participantes do estudo foram questionados quanto aos hábitos gerais (uso de tabaco/derivados e consumo de bebidas alcoólicas) e de higiene bucal praticados em sua rotina diária (número de escovações diárias e uso de fio dental). Foram excluídos da amostra os indivíduos edêntulos, em tratamento médico para neoplasias ou que estivessem em tratamento utilizando medicamentos antiinflamatórios, antibióticos, bisfosfonatos, imunossupressores ou anticonvulsivantes. Quando da necessidade de tratamento odontológico, os trabalhadores foram devidamente referenciados.

O impacto das condições bucais nas atividades diárias foi avaliado por meio do instrumento OHIP $14^{21}$. A aplicação foi realizada através de entrevista, no mesmo dia e imediatamente após o exame clínico, uma vez que sua utilização através de um formulário auto-aplicável poderia resultar em dados pouco confiáveis ${ }^{22}$. A frequência dos impactos observados foi avaliada nos últimos 12 meses e o formulário OHIP 14 é composto por sete dimensões (limitações funcionais, dor física, desconforto psicológico, incapacidade física, incapacidade psicológica, incapacidade social e invalidez). Para calcular o impacto das condições bucais na qualidade de vida utilizou-se o método padrão de cálculo do OHIP-14, com o peso específico para cada questão $0^{23}$. As seguintes pontuações foram atribuídas a cada resposta: 0 (nunca), 1 (raramente), 2 (às vezes), 3 (frequentemente) e 4 (sempre). Após pontuar cada questão o valor obtido foi multiplicado pelo peso de cada pergunta do formulário. Ao final, os valores foram somados à pontuação de todas as perguntas e os escores poderiam variar entre 0 e 28 pontos, sendo que quanto maior a pontuação obtida, maior seria a percepção do impacto avaliado pelo indivíduo ${ }^{21}$.

Os dados coletados foram tabulados e organizados com dupla entrada, em arquivos do programa Excel 2010 (Microsoft Office 2010). A apresentação foi constituída de uma parte descritiva, médias e desvio-padrão, por meio de tabelas e gráficos. Em relação à análise estatística, inicialmente foi aplicado o teste de normalidade e homogeneidade dos dados, sendo então empregado o teste estatístico apropriado. Utilizou-se teste $\mathrm{t}$ de Student independente por grupos para a análise de dados paramétricos, e teste Mann-Whitney para os dados não paramétricos. A associação entre as condições periodontais e os níveis de chumbo no sangue dos trabalhadores foi testada por meio de Odds Ratio, intervalo de confiança a 95\%, e teste Qui-Quadrado. Empregou-se a correlação linear de Pearson para avaliar a relação entre as dimensões e escores gerais do questionário OHIP-14 e níveis de chumbo no sangue e o Índice de placa. O nível de significância foi de $5 \%(\mathrm{p}<0.050)$. 


\section{RESULTADOS}

A amostra foi composta por 70 trabalhadores de uma fábrica de baterias automotivas no município de Bauru - SP, alocados nos setores de produção $(70 \%, \mathrm{n}=49)$ e administrativo $(30 \%, \mathrm{n}=21)$. A idade média encontrada foi de $33.21 \pm 9.43$ anos, sendo a idade mínima de 22 e máxima de 57 anos e $88.57 \%(n=62)$ pertenciam ao sexo masculino e $11.43 \%(n=8)$ ao sexo feminino.

Observou-se que $12.85 \%$ dos trabalhadores $(n=3)$ apresentavam hipertensão arterial sistêmica, $10 \%$ dos indivíduos $(\mathrm{n}=7)$ relataram ser tabagistas e $12.86 \%(\mathrm{n}=9)$ faziam uso regular de bebidas alcoólicas.

Quando questionados com relação aos hábitos de higiene bucal, $88.57 \%(\mathrm{n}=62)$ relataram escovar os dentes 3 ou mais vezes por dia e quase todos os trabalhadores $(97.14 \% ; n=68)$ do relataram o uso frequente de fio dental.

A média encontrada de nível de chumbo no sangue dos trabalhadores foi de $44.01 \pm 6.15 \mu \mathrm{g} / \mathrm{dL}$, sendo o valor mínimo encontrado de $33.12 \mu \mathrm{g} / \mathrm{dL}$ e máximo de 56.90 $\mu \mathrm{g} / \mathrm{dL}$ (Tabela 1). Observou-se a associação entre a função desempenhada na empresa e os níveis sanguíneos de chumbo entre os trabalhadores avaliados $(\mathrm{p}=0.000)$.

Tabela 1 - Níveis sanguíneo de chumbo e sua associação com a função desempenhada na empresa.

\begin{tabular}{lccc}
\hline & Produção & Administrativo & \\
& média \pm desvio-padrão & média \pm desvio-padrão & \\
\hline Niveis de chumbo & $46.15 \pm 5.41$ & $39.01 \pm 4.78$ & 0.000 \\
\hline Teste t de Student, para grupos independentes $(p<0.05)$.
\end{tabular}

Observou-se alta prevalência de alterações periodontais nos trabalhadores $(67.14 \% ; \mathrm{n}=47)$, sendo que o sangramento gengival esteve presente em $25.71 \%(\mathrm{n}=18)$, cálculo dentário em $67.14 \%(\mathrm{n}=47)$, bolsas rasas em $20.00 \%$, e bolsas profundas em $10.00 \%$ dos indivíduos (Figura 1).

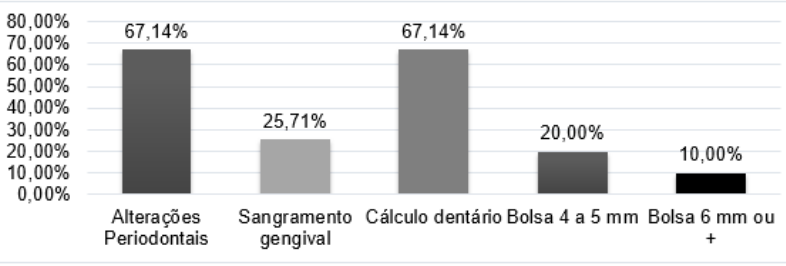

Figura 1 - Prevalência de alterações periodontais e componentes do Índice Periodontal Comunitário entre os trabalhadores avaliados.

Os indivíduos que exibiam níveis sanguíneos de chumbo $>40 \mu \mathrm{g} / \mathrm{dl}$ apresentaram 4.91 vezes mais chances de possuir sangramento gengival. A presença de cálculo e de bolsas periodontais não estiveram associados aos níveis de chumbo $(\mathrm{p}>0.05)($ Tabela 2$)$.

Tabela 2 - Associação entre os níveis de chumbo sanguíneo e as condições periodontais entre os trabalhadores avaliados.

\begin{tabular}{|c|c|c|c|c|c|c|c|c|c|}
\hline \multirow[b]{2}{*}{$\begin{array}{l}\text { Niveis de } \\
\text { chumbo }\end{array}$} & \multicolumn{3}{|c|}{ Sangramento } & \multicolumn{3}{|c|}{ Cálculo } & \multicolumn{3}{|c|}{ Bolsas } \\
\hline & OR & $\begin{array}{c}\text { IC } \\
95 \%\end{array}$ & $p$ & OR & $\begin{array}{c}\text { IC } \\
95 \%\end{array}$ & $p$ & OR & $\begin{array}{c}\text { IC } \\
95 \%\end{array}$ & $p$ \\
\hline $\begin{array}{l}\leq 40 \mu \mathrm{g} / \mathrm{dl} \\
>40 \mu \mathrm{g} / \mathrm{dl}\end{array}$ & 4.91 & $\begin{array}{l}1.51- \\
15.97\end{array}$ & 0.006 & 1.14 & $\begin{array}{l}0.40- \\
3.27\end{array}$ & 0.810 & 2.23 & $\begin{array}{l}0.64- \\
7.70\end{array}$ & 0.199 \\
\hline
\end{tabular}

OR= Odds Ratio; IC 95\%= Intervalo de confiança a 95\%; $X^{2}=$ Qui-quadrado $(p<0.05)$.

$\mathrm{O}$ índice de placa dos trabalhadores avaliados foi de $2.78 \pm 0.54$, sendo o valor mínimo encontrado de 1.43 , e o máximo de 4.02. Houve média prevalência de placa dentária entre os indivíduos avaliados. Não foi observada associação entre as condições de higiene bucal e a função desempenhada na empresa $(\mathrm{p}>0.05)$ (Tabela 3$)$.

Tabela 3 - Associação entre função desempenhada na empresa e índice de placa, em trabalhadores de uma fábrica de baterias automotivas, em Bauru - SP.

\begin{tabular}{cccc}
\hline & $\begin{array}{c}\text { Produção } \\
\text { média } \pm d p\end{array}$ & $\begin{array}{c}\text { Administrativo } \\
\text { média } \pm d p\end{array}$ & $p$ \\
\hline Índice de placa & $2.76 \pm 0.47$ & $2.83 \pm 0.69$ & 0.423 \\
\hline Teste Mann-Whitney $(\mathrm{p}<0.05)$. & &
\end{tabular}

Com relação ao impacto das condições bucais na qualidade de vida dos trabalhadores avaliados, a média dos escores gerais do questionário OHIP-14 foi $4.48 \pm 1.87$, considerado baixo impacto, variando entre 2.00 e 12.83 (Tabela 4).

Tabela 4 - Impacto das condições bucais na qualidade de vida, segundo dimensões e escore geral do questionário OHIP-14, entre os trabalhadores avaliados.

\begin{tabular}{cccc}
\hline Dimensões OPIP-14 & média \pm dp & mínimo & máximo \\
\hline Limitações funcionais & $0.69 \pm 0.50$ & 0.00 & 3.02 \\
Dor física & $0.75 \pm 0.61$ & 0.00 & 2.66 \\
Desconforto psicológico & $0.70 \pm 0.48$ & 0.00 & 1.90 \\
Incapacidade física & $0.64 \pm 0.54$ & 0.00 & 2.48 \\
\hline Incapacidade psicológica & $0.65 \pm 0.46$ & 0.00 & 2.20 \\
\hline Incapacidade social & $0.59 \pm 0.39$ & 0.00 & 1.62 \\
Invalidez & $0.47 \pm 0.45$ & 0.00 & 1.59 \\
Escore total OPIP-14 & $4.48 \pm 1.87$ & 2.00 & 12.83 \\
\hline
\end{tabular}

Não foram observadas diferenças entre as dimensões e escores gerais do questionário OHIP-14 entre os trabalhadores quanto à área de atuação na empresa avaliada (Tabela 5). 
Tabela 5 - Associação entre função desempenhada na empresa e o impacto das condições bucais na qualidade de vida, segundo dimensões e escores gerais do questionário OHIP-14, entre os trabalhadores avaliados.

\begin{tabular}{cccc}
\hline Dimensões OPIP-14 & $\begin{array}{c}\text { Produção } \\
\text { média } \pm \mathrm{dp}\end{array}$ & $\begin{array}{c}\text { Administrativo } \\
\text { média } \pm \mathrm{dp}\end{array}$ & $\boldsymbol{p}$ \\
\hline Limitações funcionais & $0.70 \pm 0.54$ & $0.64 \pm 0.39$ & 0.636 \\
\hline Dor física & $0.75 \pm 0.62$ & $0.76 \pm 0.63$ & 0.925 \\
\hline Desconforto psicológico & $0.75 \pm 0.46$ & $0.56 \pm 0.52$ & 0.131 \\
\hline Incapacidade física & $0.71 \pm 0.51$ & $0.48 \pm 0.59$ & 0.105 \\
\hline Incapacidade psicológica & $0.64 \pm 0.43$ & $0.67 \pm 0.55$ & 0.806 \\
\hline Incapacidade social & $0.59 \pm 0.40$ & $0.60 \pm 0.35$ & 0.944 \\
\hline Invalidez & $0.47 \pm 0.43$ & $0.45 \pm 0.52$ & 0.882 \\
\hline Escore total OPIP-14 & $4.61 \pm 1.83$ & $4.16 \pm 1.98$ & 0.363 \\
\hline Teste t de Student, independente por grupos $(\mathrm{p}<0.05)$. & & \\
\hline
\end{tabular}

Foi observada correlação positiva entre os níveis de chumbo presentes no sangue dos trabalhadores com a incapacidade física $(r=0.35 ; \mathrm{p}=0.003)$, e dos escores totais com o índice de placa $(\mathrm{r}=0.24 ; \mathrm{p}=0.044)$ (Tabela 6$)$.

Tabela 6 - Correlação entre os níveis de chumbo sanguíneo e o índice de Placa e as dimensões e escore geral do questionário OHIP-14, em trabalhadores de uma fábrica de baterias automotivas, em Bauru - SP.

\begin{tabular}{ccccc}
\hline & \multicolumn{2}{c}{ Niveis de Chumbo } & \multicolumn{2}{c}{ Índice de Placa } \\
& $\mathbf{r}$ & $\boldsymbol{p}$ & $\mathbf{r}$ & $\boldsymbol{p}$ \\
\hline Limitações funcionais & 0.17 & 0.143 & 0.21 & 0.078 \\
Dor física & 0.18 & 0.141 & 0.20 & 0.089 \\
& & & & \\
\hline Desconforto psicológico & 0.16 & 0.194 & 0.19 & 0.115 \\
\hline Incapacidade física & 0.35 & $\mathbf{0 . 0 0 3}$ & -0.10 & 0.388 \\
\hline Incapacidade psicológica & 0.01 & 0.939 & 0.12 & 0.310 \\
\hline Incapacidade social & -0.08 & 0.51 & 0.11 & 0.371 \\
\hline Invalidez & 0.09 & 0.467 & 0.19 & 0.114 \\
\hline Escore total OHIP-14 & 0.25 & 0.034 & 0.24 & $\mathbf{0 . 0 4 4}$ \\
\hline
\end{tabular}

\section{DISCUSSÃO}

O presente estudo evidenciou a associação entre a presença de sangramento gengival e os níveis sanguíneos de chumbo em trabalhadores de uma fábrica de baterias automotivas no município de Bauru - SP.

A amostra foi constituída predominantemente por trabalhadores do sexo masculino $(88.57 \%)$, o que pode ser atribuído ao tipo de atividade laborativa desenvolvida ${ }^{24}$ e a idade média dos trabalhadores avaliados $(33.21 \pm 9.43$ anos) se assemelha ao encontrado em uma pesquisa sobre dor orofacial entre trabalhadores do setor metalúrgico, onde a população adulta jovem foi a mais prevalente ${ }^{25}$. Em 2011, o Ministério do Trabalho e Emprego, citando levantamento da Relação Anual de Informações Sociais (RAIS) para o ano de 2009, relatou que a maior parcela dos trabalhadores ativos no Brasil esteve situada na faixa entre 30 e 39 anos, demonstrando que a amostra avaliada neste estudo pertence a um grupo de pessoas que se encontra em plena atividade laborativa e, portanto, sujeita aos acidentes e doenças causados pelas condições de trabalho ${ }^{3}$.

A prevalência de hipertensão arterial sistêmica encontrada entre os trabalhadores foi de $12.85 \%$, baixa quando comparada ao encontrado na população brasileira em geral, de $24.5 \%{ }^{26}$. Outro dado importante e que se assemelha ao encontrado previamente em indivíduos adultos, foi a prevalência do etilismo entre os trabalhadores $12.86 \%$ e o consumo de tabaco entre os trabalhadores avaliados ${ }^{26}$.

Apesar dos níveis sanguíneos de chumbo dos trabalhadores encontrarem-se abaixo do Índice Máximo Biológico Permitido, observou-se que função desempenhada contribuiu para níveis mais elevados de chumbo no sangue, estando os indivíduos alocados no setor de produção mais expostos. Entre 1995 e 1996, níveis sanguíneos de chumbo de trabalhadores do setor metalúrgico de Bauru - SP foram avaliados a fim de se observar a presença de contaminação entre os indivíduos ${ }^{27}$. Os autores confirmaram a contaminação em $18.10 \%$ da amostra avaliada, sendo que nenhum dos indivíduos avaliados apresentou valores de nível de chumbo no sangue superiores ao Índice Máximo Biológico Permitido. Entretanto, observou-se diferença significativa entre os níveis de chumbo e a função desempenhada na empresa.

O chumbo é um elemento que não é metabolizado pelo organismo, parte dele é excretado e parte dele é armazenada em tecido duro e tecido mole. Dependendo da quantidade de chumbo que se encontra no organismo, podem ocorrer desde náuseas até doenças neurológicas como o saturnismo. Os principais efeitos acontecem a nível hematológico, renal, digestivo e no sistema nervoso central. Há evidencias de que o chumbo tenha também ação hemolítica, através da indução de alterações nas propriedades antioxidantes da membrana eritrocitária e da hemoglobina, consequentemente podendo surgir a anemia hemolítica microcítica, hipocrômica leve $\mathrm{e}^{27}$.

Trabalhadores de fábricas de bateria estão expostos a névoas ácidas, decorrente do manuseio de materiais potencialmente tóxicos, dentre os quais o chumbo. Dados obtidos em estudos evidenciaram a associação que pode existir entre exposição ocupacional e a ocorrência de problemas bucais em trabalhadores deste setor da indústria ${ }^{28-29}$.

Pelo fato das condições do ambiente e de determinados tipos de trabalho pode influenciar a saúde dos tecidos periodontais, alguns estudos investigaram a exposição a produtos ácidos no ambiente de trabalho como sendo um fator de risco ao desenvolvimento de alterações no periodonto e observaram associação positiva entre essa exposição e problemas ocorridos nos tecidos de sustentação como sangramento gengival e bolsas periodontais ${ }^{29-31}$.

A prevalência de alterações periodontais entre os trabalhadores avaliados neste foi considerada alta (67.14\%), 
porém a condição que mais contribuiu para este achado foi a presença de cálculo dentário (67.14\%), seguido pelo sangramento gengival $(25.71 \%)$ e bolsas periodontais rasas $(20.00 \%)$ e profundas $(10.00 \%)$. Em um estudo sobre condições bucais de trabalhadores de uma indústria metalúrgica, de Barros e colaboradores (2012) ${ }^{24}$ observaram que $30.22 \%$ dos indivíduos relataram a presença de sangramento gengival, e ao exame clínico constataram a presença de cálculo dentário em $55.00 \%$ dos indivíduos.

Comparando-se os resultados encontrados no presente estudo aos dados provenientes do Levantamento Nacional de Saúde Bucal SB Brasil 2010, para a mesma faixa etária, podemos observar que a condição periodontal dos trabalhadores avaliados foi mais favorável do que a da média nacional da população que se encontra em atividade laborativa para todas as categorias deste índice, onde $32.86 \%$ dos indivíduos avaliados apresentavam-se livres de alterações periodontais, contra apenas $17.80 \%$ no levantamento nacional. Contudo, quando comparamos os resultados com o levantamento SB Brasil 2003, observouse que a condição periodontal piorou de 2003 para 2010 , fato este que pode estar associado com o envelhecimento da população brasileira ${ }^{16,32}$.

Os trabalhadores avaliados relataram alta frequência de escovações e uso de fio dental, porém o percentual de cálculo dentário (67.14\%) e índice de placa (2.78 \pm 0.54$)$ encontrado denotam a pobre condição de higiene bucal destes indivíduos. É provável que os trabalhadores não estejam fazendo sua higiene bucal de forma adequada e necessitem de maior atenção na área preventiva do que curativa ${ }^{33}$.

Embora as diferenças nas necessidades em saúde não sejam eliminadas apenas com o uso de serviços de saúde, é inegável que o acesso a serviços de qualidade pode amenizar condições desfavoráveis de saúde em populações $^{34}$. Em levantamento previamente realizado com trabalhadores do mesmo setor produtivo, os autores observaram que os trabalhadores possuíam acesso a assistência odontológica oferecida pelas empresas, onde os mesmos puderam receber tratamento para procedimentos menos complexos, contribuindo para as condições favoráveis encontradas ${ }^{33}$. Deve-se ressaltar, que os trabalhadores avaliados neste estudo possuíam acesso a serviços de saúde bucal por meio de convênios, oferecidos como benefício aos funcionários, o que não demonstrou contribuir para a presença de uma condição periodontal e de higiene bucal adequada.

Foi observada correlação positiva entre os níveis de chumbo no sangue dos trabalhadores com a incapacidade física, ou seja, quanto maior o nível de chumbo maior a percepção de incapacidade - na mastigação, dor ou desconforto. Segundo um estudo realizado na Espanha ${ }^{35}$, existe relação entre a quantidade do chumbo e patologia periodontal. Foram analisados 220 dentes, sendo 41 deles de adultos entre 30 a 59 anos, os dentes foram submetidos a análise da quantidade de chumbo neles presentes e foi evidenciado que nos dentes que apresentavam patologias periodontais os níveis de chumbo eram maiores. O biofilme dental apresenta-se como agente determinante de cárie dentária e periodontopatias, as quais se caracterizam como os principais problemas de saúde bucal ${ }^{36}$. A doença periodontal pode levar a perda dos dentes, podendo causar um impacto negativo na qualidade de vida do indivíduo.

As possíveis limitações deste estudo estiveram relacionadas ao índice empregado para a avaliação da condição periodontal dos trabalhadores, o IPC, que é recomendado para utilização em estudos epidemiológicos, pois é de fácil e rápida execução, porém o mesmo poderia sub ou superestimar a prevalência das alterações encontradas; outro fator a ser considerado diz respeito ao tamanho da amostra $(n=70)$, representativa apenas para os trabalhadores da empresa pesquisada. Estudos futuros com amostras mais amplas, desenhos longitudinais e avaliação periodontal completa (todos os elementos dentários) poderiam auxiliar na compreensão da associação entre a exposição ao chumbo no ambiente de trabalho e as alterações periodontais encontradas.

\section{CONCLUSÃO}

A partir do exposto pode-se concluir que nenhum dos trabalhadores avaliados neste estudo apresentou níveis sanguíneos de chumbo acima do Índice Máximo Biológico Permitido (IMBP) e a prevalência de alterações periodontais encontrada foi alta, porém as alterações mais proeminentes estiveram relacionadas à pobre higiene bucal dos trabalhadores. Embora os trabalhadores tenham relatado alta frequência de escovações e uso de fio dental, pode-se observar grande acúmulo de placa e cálculo dentário, evidenciando a necessidade de adoção de medidas terapêuticas, preventivas e educativas visando a melhora das condições bucais avaliadas. Os trabalhadores que atuavam no setor de produção apresentaram níveis sanguíneos de chumbo significativamente maiores do que os trabalhadores do setor administrativo na empresa avaliada, e os níveis mais elevados de chumbo estiveram associados à presença de sangramento gengival. Os trabalhadores apresentaram baixa percepção de impacto das condições bucais na qualidade de vida, porém esta pode ser correlacionada positivamente com a pobre higiene bucal, e os níveis de chumbo foram correlacionados ao domínio incapacidade física, o que pode acarretar em prejuízos às condições bucais dos trabalhadores a longo prazo. 


\section{AGRADECIMENTOS}

Os autores agradecem à Fundação de Amparo à Pesquisa do Estado de São Paulo - FAPESP, pelo apoio e fomento a esta pesquisa (Processo ${ }^{\circ}$ 2013/26103-0).

\section{REFERÊNCIAS}

1. Conselho Federal de Odontologia. Resolução $\mathrm{CFO}^{\circ}{ }^{\circ} 63$, de 19 de abril de 2005, atualizado em 5 de julho de 2006. Rio de Janeiro: CFO; 2006.

2. De Carli BMG. Doenças ocupacionais com manifestações bucais. Odonto. 2013;20(40):49-55.

3. Brasil. Ministério do Trabalho e Emprego. Relação anual de informações sociais (RAIS). Brasília: Ministério do Trabalho e Emprego; 2011.

4. Minozzo R, Deimling LI, Gigante LP, Santos-Mello R. Micronuclei in peripheral blood lymphocytes of workers exposed to lead. Mutat Res. 2004;565(1):53-60.

5. Moreira FR, Moreira JC. Lead kinetics in human body and its significance to health. Cien Saude Colet. 2004;9(1):167.

6. Pounds JG, Long J, Rosen JF. Cellular and molecular toxicity of lead in bone. Environ Health Perspect. 1991;91:17-32.

7. Silbergeld EK et al. Lead in bone: storage site, exposure source, and target organ. Neurotoxicology. 1993;14(2-3):225-36.

8. Pinkerton LE, Biagini RE, Ward EM, Hull RD, Deddens JA, Boeniger MF, et al. Immunologic findings among lead-exposed workers. Am J Ind Med. 1998;33(4):400-8.

9. Pineda-Zavaleta AP. Nitric oxide and superoxide anion production in monocytes from children exposed to arsenic and lead in region Lagunera, Mexico. Toxicol Appl Pharmacol. 2004;198(3):283-90.

10. Offenbacher S. Periodontal diseases: pathogenesis. Ann Periodontol. 1996;1:821-78.

11. Saraiva MCP. Lead exposureandperiodontitis in US adults. J Perio Res. 2007;42(1):45-52.

12. Esteves RC. Manifestações bucais das doenças profissionais. Rev Bras Saude Ocup. 1982;10(40):56-8.

13. Agency for Toxic Substances and Disease Registry (US). Toxicological profile for lead. Atlanta: Public Health Service; 2007.

14. Brasil. Ministério do Trabalho e Emprego. Norma Regulamentadora n 7: nota técnica. Brasília: Ministério do Trabalho e Emprego; 1996.

15. Minozzo R. Prevalence of anemia in workers with work-related exposure to lead. Rev Bras Hematol Hemoter. 2009;31(2):94-7.

16. Brasil. Ministério da Saúde. SBBrasil 2010. Pesquisa Nacional de Saúde Bucal: resultados principais. Brasília: Ministério da Saúde; 2012.

17. World Health Organization. Oral health surveys: basic methods. 5th ed. Geneva: WHO; 2013.

18. Landis JR, Koch GG. Biometrics. The measurement of observer agreement for categorical data. Biometrics.1977;33(1):159-74.
19. Turesky S, Gilmore ND, Glickman I. Reduced plaque formation by the chloromethyl analogue of victamine C. J Periodontol. 1970;41(1):41-3.

20. Quigley GA, Hein JW. Comparative cleansing efficiency of manual and power brushing. J Am Dent Assoc. 1962;65:26-9.

21. Slade GD. Derivation and validation of a short-form oral health impact profile. Community Dent Oral Epidemiol. 1997;25(4):284-90.

22. Oliveira BH, Nadanovsky P. Psychometric properties of the Brazilian version of the oral health impact profile-short form. Community Dent Oral Epidemiol. 2005;33(4):307-14.

23. Allen PF, Locker D. Do item weights matter? An assessment using the oral health impact profile. Community Dent Health. 1997;14(3):133-8.

24. Barros ACM, Schmidt CM, Marote IAA, Queluz DP. Perfil da saúde bucal de trabalhadores na indústria metalúrgica. Odonto. 2012;20(40): 73-87.

25. Lacerda JT, Traebert J, Zambenedetti ML. Dor orofacial e absenteísmo em trabalhadores da indústria metalúrgica e mecânica. Saude Soc. 2008;17:182-91

26. Brasil. Ministério da Saúde. Vigitel Brasil 2019: vigilância de fatores de risco e proteção para doenças crônicas por inquérito telefônico. Brasília: Ministério da Saúde; 2020.

27. Demarchi ACCO, Souza LCD, Santos LS, Menezes ML. Avaliação da exposição ao chumbo em trabalhadores das indústrias de Bauru. Salusvita. 1999;18(1):27-36.

28. Petersen PE, Gormsen C. Oral conditions among german battery factory workers. Community Dent. Oral Epidemiol. 1991;19(2):104-6.

29. Almeida TF, Vianna MIP, Santana V, Gomes Filho IS. Occupational exposure to acid mists and periodontal attachment loss. Cad Saude Publica. 2008;24(3):495-502.

30. Tuominen M. L. Occurrence of periodontal pockets and oral soft tissue lesions in relation to sulfuric acid fumes in the working environment. Acta Odontol Scand. 1991;49(5):261-6.

31. Amin WA, Al-Omoush SA, Hattab FN. Oral health status of workers exposed to acid fumes in phosphate and battery industries in Jordan. Int Dent J. 2001;51(3):169-74.

32. Gaião LR, Almeida MEL, Heukelbach J. Perfil epidemiológico da cárie dentária, doença periodontal, uso e necessidade de prótese em idosos residentes em uma instituição na cidade de Fortaleza, Ceará. Rev Bras Epidemiol. 2005;8(3):316-23.

33. Aznar FDC, Calzavara B, Freitas AR, Aznar FDC, Sales-Peres SHC, Sales-Peres A. Condição bucal de trabalhadores de fábricas de baterias e uso de serviços odontológicos. Rev Bras Med Trab. 2016;14(2):127-33.

34. Pinheiro RS, Torres TZG. Uso de serviços odontológicos entre os Estados do Brasil. Cienc Saude Coletiva. 2006;11(4):999-1010.

35. Gil F, Pérez ML, Facio A, Villanueva E, Tojo R, Gil A. Dental lead levels in the galician population, Spain. Sci Total Environ. 1994;156(2):145-50.

36. Toassi RFC, Petry PC. Motivação no controle do biofilme dental e sangramento gengival em escolares. Rev Saude Publica. 2002;36:634-7. 